\title{
Zervikales Wurzelkompressionssyndrom
}

\author{
Heinrich Böhm, Reinhard Bschorer
}

\section{Ausgangssituation}

Eine damals 34-jährige Patientin wurde wegen eines trotz aller konservativen Maßnahmen therapierefraktären zervikalen Wurzelkompressionssyndroms in der Etage C5 - C6 von ventral fusioniert. Wegen erneuter Beschwerden im Dermatom C8 erfolgte eine ventrale Nachoperation mit Verlängerung der Fusion auf Th1 und Instrumentation mit einer winkelstabilen Platte. Diese lockert rasch nach kaudal aus, parallel steigen die Infektparameter. In der Bildgebung zeigt sich mit Sicherheit eine Spondylodiszitis C7 - Th1, mit Wahrscheinlichkeit auch in der Etage C6-C7.

\section{Befund}

Septischer Allgemeinzustand, stark schmerzhafte Bewegungseinschränkung und Erschütterungsempfindlichkeit der Halswirbelsäule. Keine Schluckbeschwerden.

\section{Röntgen}

Kyphosierung bei knöchernen Defekten und Sklerosierungen an der Grundplatte C7 sowie Deckplatte Th1, geringe Destruktionen bei C6-C7, erfolgreiche Fusion $\mathrm{C} 5-\mathrm{C} 6$, es liegt eine ventrale winkelstabile Platte (Abb.1), MRT bei Stahlimplantat nicht verwertbar.

\section{Weiterer Verlauf}

Es wird ein radikales ventrales Débridement mittels Korporektomie C6 und C7 durchgeführt, verbunden mit Spongiosainterposition, lokaler Antibiose und dorsaler Plattenspondylodese (Abb.2). Daraufhin rascher Rückgang der Infektsymptomatik. Im weiteren Verlauf aber Entwicklung einer progredienten zervi-

OP-JOURNAL 2008; 24: 196-197

(c) Georg Thieme Verlag KG Stuttgart • New York DOI 10.1055/s-2008-1039144

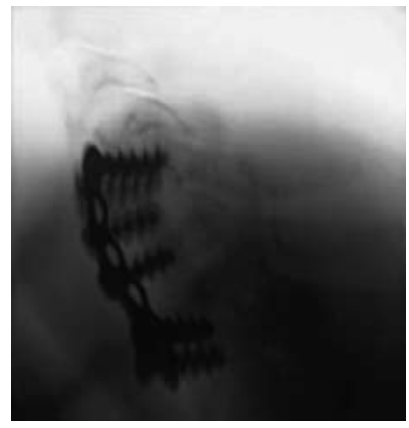

Abb. 1 latrogene Spondylodiszitis C7-Th1 bei liegender ventraler winkelstabiler Platte. Fusion C5 - C6 knöchern durchbaut, C6 C7 radiologisch suspekt. MRT wegen Stahlimplantat nicht verwertbar.

kalen Myelopathie sowie bei C7 und C8 linksseitig radikulärer sensibler und motorischer Ausfälle. Als Ursache für die neurogenen Ausfälle wird ein Einsinken des Harms-Korbes mit konsekutiver Kyphosierung der Wirbelsäule festgestellt.

Ein erneuter ventraler Eingriff an der Halswirbelsäule wird unvermeidlich zur Myelondekompression, Stellungskorrektur und Restabilisierung. Dabei muss kranial und kaudal ein weiteres Bewegungssegment geopfert werden, sodass die anschließende Versteifungsstrecke von C4 bis Th2 reicht. Die Rekon- struktion der vorderen Säule wird kombiniert mit einer dorsalen Plattenspondylodese (Abb.3). Nach diesem Eingriff bildet sich die Tetraparese vollständig zurück, aber es kommt zu massiven Dysphagien. Bereits geringe Essensmengen lösen massiven Hustenreiz aus. Ösophagusbreischluck und Ösophagoskopie bestätigen den Verdacht einer Ösophaschließlich durch Entfernung des ventralen Implantats und ein radikales vorderes Débridement bis zur Dura. Nach Schaffung gut durchbluteter Ankerpunkte an den Endwirbeln des Defekts wird ein Muskel-Knochen-Komposittransplantat aus Fibula und zwei Dritteln des M. flexor hallucis longus transferiert und an den Thyreoidalgefäßen reanastomosiert. Bei sicherer dorsaler Stabilisierung kann die Fibula in Pressfit-Technik zwischen C4 und Th2 eingefalzt werden. (Abb.5) Relevanteste Aspekte der Nachbehandlung sind die äußere Immobilisierung in einer HaloWeste für 3 Monate und die Ernährung durch eine PEG für 3 Wochen.

\section{Ergebnis}

Die Ösophagusfistel war nach 3 Wochen geschlossen, worauf der Patient auf orale Ernährung umgestellt werden konnte und symptomfrei blieb. Parallel waren die Entzündungsparameter bereits nach 2 Wochen wieder im Normalgusfistel (Abb.4). Die Sanierung gelingt

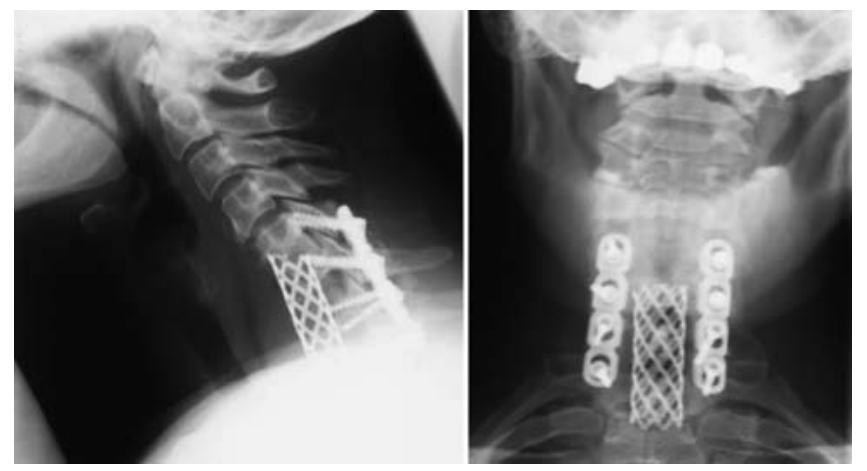

Abb. 2 Nach Korporektomie $\mathrm{C} 6$ und $C 7$ ventrale Abstützung mittels eines mit Eigenspongiosa gefüllten HarmsKorbes. Dorsale Stabilisierung und Spondylodese C5-Th1 Anschließend Ausheilen des Infekts. 

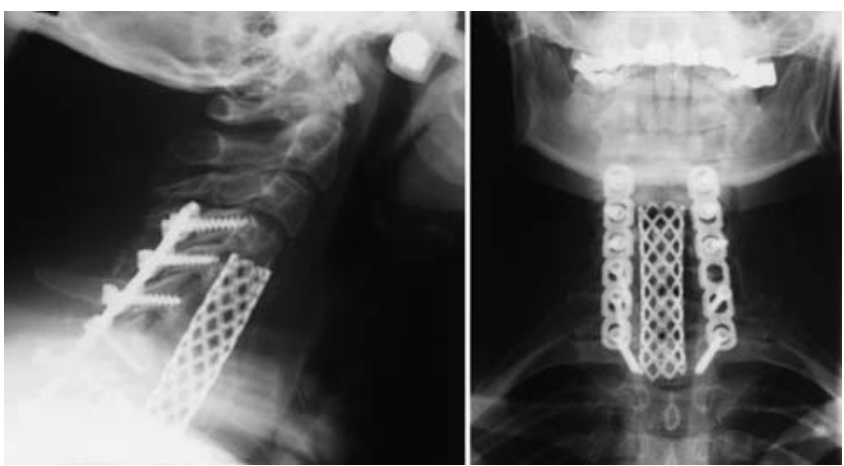

Abb. 3 Nach zunehmender Fehlstellung Auftreten einer rasch progredienten Tetraparese bei Rückenmarkkompression sowie foraminaler Kompression. Erforderlich wird die Stellungskorrektur und direkte Dekompression mittels Korporektomie C5 und Th1: Die wegen Infekt oder Rückenmarkkompression korporektomierte Strecke bemisst jetzt 4 Wirbelkörper.

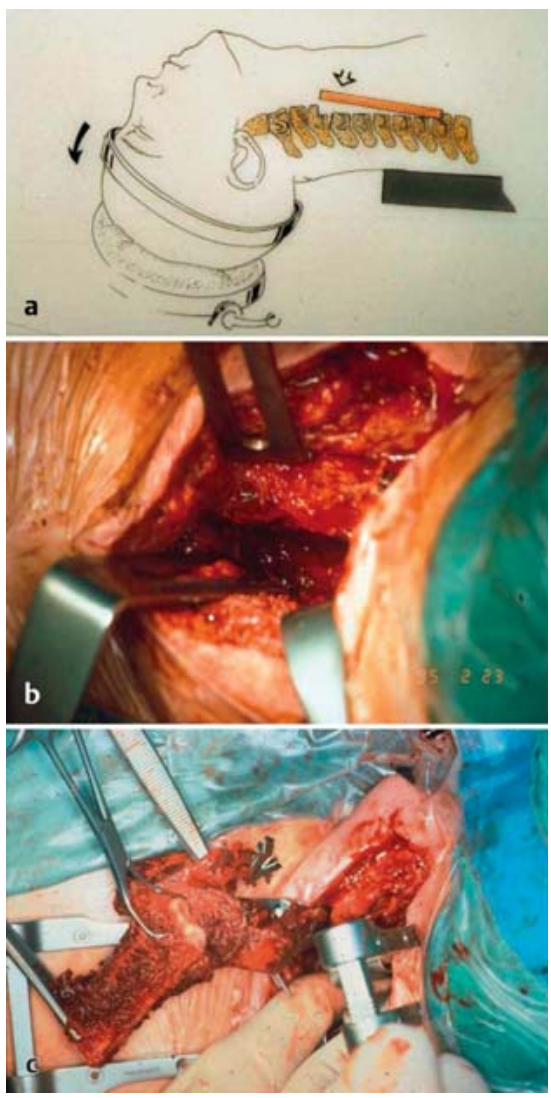

Abb. $\mathbf{5 a}$ bis c a Schematische Darstellung der Rekonstruktion der vorderen Säule von C4-Th2. b Intraoperativer Situs nach Implantatentfernung, Débridement und Duradekompression. c Transplantat mit Fibula, Extensor hallucis und Gefäßen (durch Clips markiert).

bereich und blieben es über Jahre. Die Verlaufskontrollen der Bildgebung zeigten ein sehr rasches Einwachsen der Fibula und stabile mechanische Verhältnisse über mehr als 10 Jahre (Abb.6). Von der Tetraparese blieben keine Folgen zurück; wegen Dysästhesien im

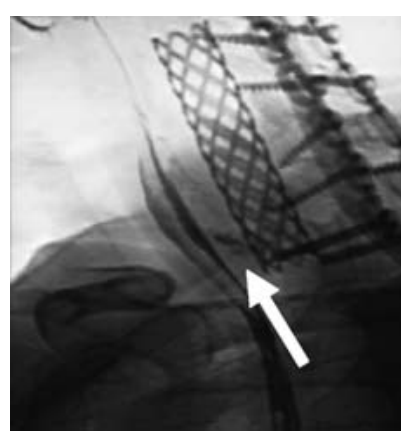

Abb. 4 Ösophagusbreischluck zeigt Extravasat in Richtung des kaudalen Endes des Harms-Korbes ziehend.
C8-Bereich links entfernten wir auf Wunsch der Patientin die linke Platte, was jedoch keine Änderung der Beschwerden bewirken konnte.

\section{Fazit}

Von den Weichteilen im Zugangsbereich zur vorderen Halswirbelsäule ist der Ösophagus mit Abstand die empfindlichste Struktur. Zur Schonung des Ösophagus sollten die Wundsperrer häufig umgesetzt, nach Voroperationen am besten zulasten von Wundhaken gar nicht verwendet werden. Nach ernsthaften ventralen Komplikationen sollte immer die dorsale mechanische Stabilisierung erwogen werden, um einen allfälligen vorderen Reeingriff so klein und implantatarm wie möglich zu halten. Wiewohl sehr ressourcenaufwendig bietet dieses Verfahren, vor allem auch wegen der Verbesserung der örtlichen Blutungsverhältnisse durch den M. extensor hallucis, sehr gute Möglichkeiten, bei prekären Situationen wie Vorbestrahlung oder Infektfolgen eine biologische Lösung und damit ein gutes Langzeitergebnis zu erzielen.

\section{Prof. Dr. med. Heinrich Böhm} Chefarzt

Klinik für Orthopädie, Wirbelsäulenchirurgie und Querschnittgelähmte Zentralklinik Bad Berka GmbH Robert-Koch-Allee 9 99437 Bad Berka

Prof. Dr. Dr. med. Reinhard Bschorer Facharzt für Mund-, Kiefer- und Gesichtschirurgie

Klinik für Mund-, Kiefer- und Gesichtschirurgie

Helios Klinikum Schwerin

Wismarsche Straße 393-397 19055 Schwerin

E-Mail: ort@zentralklinik-bad-berka.de 